\title{
NOVAS COMBINAÇÕES EM MOLDENHAWERA SCHRAD. E CRATYLIA MART. EX BENTH. E UMA NOVA VARIEDADE DE ZORNIA BRASILIENSIS VOG. (LEGUMINOSAE).
}

\author{
LUCIANO PAGANUCCI DE QUEIROZ
}

Universidade Estadual de Feira de Santana, Depto. de Ciências Biológicas, Km 03 BR 116, 44031-460, Feira de Santana, BA, Brasil.

\begin{abstract}
New combinations in Moldenhatvera Schrad. and Cratylia Mart. ex Benth., and a new variety of Zornia brasiliensis Vog. (Leguminosae). As a result of taxonomic studies of different Leguminosae groups two new combinations are proposed: Moldenhatvera emarginata (Sprengel) L.P.Queiroz \& R.Allkin (tribe Caesalpinieae) and Cratylia intermedia (Hassler) L.P.Queiroz \& R.Monteiro (tribe Phaseoleae). It is described Zornia brasiliensis var. adenocarpa L.P.Queiroz, var.nov. Three species of Moldenhazera (M. cuprea Pohl, M. nitida H.S.Irwin \& Arroyo and M. riedelii Yakovlev) are treated as synonyms of $M$. emarginata.

Resumo - (Novas combinações em Moldenhatwera Schrad. e Cratylia Mart. ex Benth. e uma nova variedade de Zornia brasiliensis Vog. (Leguminosae). Como resultado de estudos taxonômicos em diferentes grupos de Leguminosae são propostas duas novas combinações: Moldenhatvera emarginata (Sprengel) L.P.Queiroz \& R.Allkin (tribo Caesalpinieae) e Cratylia intermedia (Hassler) L.P.Queiroz \& R.Monteiro (tribo Phaseoleae). É descrita Zornia brasiliensis var. adenocarpa L.P.Queiroz, var.nov. Três espécies de Moldenhazvera (M. cuprea Pohl, M. nitida H.S.Irwin \& Arroyo e M. riedelii Yakovlev) são tratadas como sinônimos de M. emarginata.
\end{abstract}

Key words: Leguminosae, Cratylia, Moldenhawera, Zornia.

\section{Introdução}

Estudos taxonômicos em diferentes grupos de Leguminosae brasileiras, atualmente sendo conduzidos pelo autor, têm resultado na necessidade de se proceder alterações nomenclaturais e descrição de novos táxons. Ao mesmo tempo, estudos florísticos estão sendo desenvolvidos com as Leguminosae da região de Grão-Mogol (estado de Minas Gerais) e do estado de São Paulo. Este trabalho tem, então, a finalidade de validar alguns nomes e descrever um novo táxon, os quais serão usados nas versões finais destas floras.

Moldenhawera emarginata (Sprengel) L.P.Queiroz \& R.Allkin, comb. nov.

Basiônimo: Cubaea emarginata Sprengel, Syst. veg. 2: 91, 1825. Neotypus "Brasilia, inter frutices ad Tapanhoacanga cap. Min. Ger." Pohl (neotypus, aqui designado: $\mathrm{K}$ !; isoneotypi $\mathrm{BR}$ !, W; foto do neotipo: HUEFS !) Moldenhawera cuprea Pohl, Pl. Bras. Ic. 2: 91, tab. 160, 1830, nom. illeg.

Tachigali emarginata (Sprengel) G.Don, Gen. hist. 2: 436, 1832.

Moldenhauera nitida H.S.Irwin \& Arroyo, Brittonia 26 (3): 266, 1974. Typus Brasil, "Minas Gerais, Serra do Espinhaço, ca. $25 \mathrm{Km}$ NE Diamantina on road to Medanha, 30.i.1969, H.S.Irwin, R. Souza Ev S.F.Fonseca
22902 (holotypus UB !; isotypi C, F !, GH, IAN, K !, MO !, NY !, P, SP !, US !), syn. nov.

Moldenhawera riedelii Yakovlev, Bot. Zhurn. 60 (2): 219, 1975. Typus Brasil, Minas Gerais, "In montib. altis S. do Jiquitinhonha", xii.1824, Riedel 1253(holotypus LE!, isolypi LE !, K !; foto do holotipo: HUEFS !), syn. nov.

As coleções de Moldenhawera procedentes do norte do estado de Minas Gerais têm sido tradicionalmente identificadas como $M$. cuprea, seguindo o tratamento dado por Bentham (1870) na "Flora Brasiliensis". Pohl (1830), ao descrever $M$. cuprea, fez referência no protólogo a Cubaea emarginata, idenficando-a com uma espécie previamente publicada. De acordo com as regras vigentes da nomenclatura botânica (Art. 52; Greuter et al. 1994) o binômio M. cuprea, um nome supérfluo para o táxon, não pode ser considerado um nome legítimo.

Sprengel (1825) descreveu Cubaea emarginata baseado em um espécime coletado por F. Sellow. Este espécime encontra-se perdido, tendo sido provavelmente destruído no incêndio do herbário de Berlim (B) na Segunda Guerra Mundial (Hiepko 1987). Nenhuma duplicata foi encontrada nos principais herbários europeus e norte-americanos. No entanto, a descrição apresentada por Sprengel, apesar de resumida, enfoca as principais características diagnósticas desta espécie. Desta forma, considera-se estes táxons como co-específicos e o espéci- 
me Pohl "adl Tapanhoacanga cap. Min. Ger." é selecionado como neótipo, já que, dos espécimes disponíveis, é o que mais se aproxima da diagnose contida no protólogo de Cubaea emarginata, tentando, assim, preservar o uso original pretendido pelo autor (Sprengel, 1825), de acordo com a recomendação do código de nomenclatura botânica (Art. 9, Rec. 9B; Greuter et al. 1994). Ressaltese ainda que Sellow realizou coletas na mesma localidade em que Pohl colheu o espécime tipo de $M$. cuprea (Herter \& Rambo, 1953), sendo possível que os espécimes coletados por estes dois naturalistas fossem provenientes de uma mesma localidade geral.

O exame dos tipos de $M$. nitida e $M$. riedelii, além de coleções recentes de Moldenhawera procedentes do norte de Minas Gerais, mostrou que estes táxons incluem-se na variação de $M$. emarginata, sendo aqui tratados como sinônimos.

Cratylia intermedia (Hassler) L.P.Queiroz \& R.Monteiro, comb. et stat. nov.

Basiônimo: Cratylia floribunda Benth. var. intermedia Hassler, Ann. Cons. Jard. Bot. Genève 21: 219, 1919. Typus: Argentina, prov. Missiones, "in sylvis ad cataractas fluminis Yguazu”, Osten 8187 (holotypus G !).

Hassler (1919), ao descrever este táxon, considerouo como uma variedade de $C$. floribunda (presentemente C. argentea (Desv.) O.Kuntze; Queiroz 1991). No entanto, esta planta apresenta folíolos mais coriáceos, pseudoracemos mais curtos e robustos e flores maiores do que $C$. argentea. Além disto, possui inflorescência ereta com nodosidades capitadas e sésseis, enquanto $C$. $a r$ gentea apresenta inflorescência patente a pêndula com nodosidades clavadas e pedunculadas.

Além das características morfológicas pode-se notar que as áreas de distribuição de C. argentea e C. intermedia são disjuntas. $C$. intermedia é restrita às bacias dos rios Paraná e Iguaçu, sendo conhecida apenas do estado do Paraná, sul do estado de São Paulo (Brasil) e extremo nordeste da província de Missiones (Argentina). Já $C$. argentea, apesar de amplamente distribuída do oeste do Peru e Bolívia até o estado do Ceará, encontra seu limite meridional a ca. $18^{\circ} \mathrm{S}$ no sul do estado de Mato Grosso (Queiroz \& Coradin 1996).

Desta forma, tanto as características morfológicas como a distribuição geográfica justificam a atribuição de status específico distinto a este táxon.

Zornia brasiliensis Vogel var. adenocarpa L.P.Queiroz, var. nov.

Lomenti articuli concavi ut in varietas typica sed glanduilis patelliformibus praesentia differt.
Typus: Brasil, estado de Minas Gerais, Grão-Mogol: vale do rio Itacambiruçu ao longo da estrada para Cristália, $16^{\circ} 30^{\prime} \mathrm{S} 42^{\circ} 55^{\prime} \mathrm{W}$, ca. $750 \mathrm{~m}$ alt. 10.xii.1989, P.T.Sano, T.R.S.Silva, J.R.Pirani, A.Freire-Fierros CFCR 12384 (holotypus SPF !; isotypi HUEFS !, K).

Paratypus: Brasil, estado de Minas Gerais, Grão-

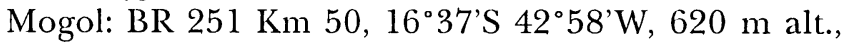
18.ii.1989, T.B.Cavalcanti, J.Kirkbride, W.Roall, G.P.da Silva 268 (CEN, SPF !).

Subarbusto prostrado $30-40 \mathrm{~cm}$ alt., com ramos vilosos. Estípulas peltadas, lanceoladas, $8-10$ x 2-3 mm. Folhas palmadamente compostas, 4-folioladas, pecíolo 0,7-1 cm compr., folíolos obovais a oblanceolados, ápice arredondado, base cuneada, os distais mais estreitos e agudos, densamente pontuados, pilosos a vilosos, 1,52,9 x 0,4-0,8 cm, peciólulo 1-2 mm compr. Espigas axilares $3-5 \mathrm{~cm}$ compr., brácteas ovais, falcadas, pubescentes, pontuadas, 7-8 x 2-3 mm. Cálice com tubo campanulado ca. 1,5 $\mathrm{mm}$ compr., lacínio superior emarginado 1,5-2 $\mathrm{mm}$ compr., lacínios laterais obsoletos, denticulados, lacínio inferior subulado ca. $1 \mathrm{~mm}$ compr., pétalas amarelas, lâmina do estandarte orbicular 89 x 9-10 mm. Lomento 5-8 mm compr., 3-5-articulado; artículos ca. 2 x 1,8-2 mm, hemiorbiculares, sinuados, reticulados, côncavos no dorso, esparsamente pilosos e com cerdas esparsas de ca. $0,5 \mathrm{~mm}$.

Mohlenbrock (1961), ao revisar o gênero Zornia, considerou Z $Z$. brasiliensis distinta das demais espécies tetrafolioladas especialmente pelos artículos do lomento dorsalmente côncavos e desprovidos de glândulas. Autores subseqüentes adotaram esta conceituação, embora Lewis (1987) tenha chamado a atenção para a complexa história nomenclatural desta espécie e sua semelhança com $Z$. marajoara Huber.

A ocorrência de lomentos glandulosos em uma população de $Z$. brasiliensis tende a tornar ainda mais complexa a taxonomia deste grupo. No entanto, a forma dos artículos revela-se um bom caráter diagnóstico para este táxon, já que é a única Zornia tetrafoliolada a apresentar o dorso côncavo, semelhante ao que é mais comumente observado em espécies de Stylosanthes. Desta forma, utiliza-se este caráter, mais do que a presença/ausência de glândulas, como diagnóstico para esta espécie. O epíteto usado para esta nova variedade ressalta a ocorrência de artículos glandulosos, condição distinta da encontrada na variedade típica.

Z. brasiliensis var. adenocarpa é conhecida exclusivamente da serra de Grão-Mogol, no norte do estado

' CFCR = Coleção para a Flora dos Campos Rupestres 
de Minas Gerais, ocorrendo entre 600 e $750 \mathrm{~m}$ alt., florescendo e frutificando em dezembro e fevereiro.

\section{Agradecimentos}

O autor agradece ao Dr. José R. Pirani, coordenador da Flora de Grão-Mogol, e à Dra. Ana M. G. Azevedo-Tozzi, coordenadora da família Leguminosae para a Flora Fanerogâmica do estado de São Paulo, pelo convite para participar destes estudos florísticos, e aos curadores dos herbários referidos no texto pelo empréstimo dos tipos nomenclaturais. Este trabalho foi fïnanciado pela CAPES, através de uma bolsa de mestrado que possibilitou o desenvolvimento da revisão taxonômica de Cratylia, pelo British Council, Fundação Botânica Margaret Mee e Universidade Estadual de Feira de Santana que possibilitaram uma visita do autor ao Royal Botanic Gardens Kew onde foi desenvolvida a revisão taxonômica de Moldenhawera.

\section{Referências}

BENTHAM, G. 1870. Leguminosae. In C.F.P.Martius (ed.) Flora brasiliensis 15(2):1-528.
GREUTER, W., BARRIE, F.R., BURDET, H.M., CHALONER, W.G., DEMOULIN, V., HAWKSWORTH, D.L., JØRGENSEN, P.M., NICOLSON, D.H., SILVA, P.C., TREHANE, P., McNEILL, J. (eds.) 1994. International Code of Botanical Nomenclature (Tokyo Code) adopted by the Fifteenth International Botanical Congress, Yokohama, August-September 1993. Regnum Veg. 131.

HASSLER, E. 1919. Primitiae Missionum Argentinarum. I. Annuarie Conserv. Jard. Bot. Genètue 21:217-220.

HERTER, W.G. \& RAMBO, B. 1953. Nas pegadas dos naturalistas Sellow e Saint-Hilaire. Revista Sudamer. Bot. 10 (3): 61 - 98.

HIEPKO, P. 1987. The collections of the Botanical Museum BerlinDahlem (B) and their history. Englera 7:219-252.

LEWIS, G.P. 1987. Legumes of Bahia. Royal Botanic Gardens Kew, $369 \mathrm{pP}$.

MOHLENBROCK, R. 1961. A monograph of the Leguminous genus Zornia. Webbia 16 (1):1-141.

POHL, J.E. 1830. Plantarum Brasiliae icones et descriptiones vol 2, $153 \mathrm{pP}$.

QUEIROZ, L.P.de 1991. O gênero Cratylia Martius ex Bentham (Leguminosae: Papilionoideae: Phaseoleae): revisäo taxonômica e aspectos biológicos. Dissertação de Mestrado Univ.Est.de Campinas, 168 pp.

QUEIROZ, L.P.de \& CORADIN, L. 1996. Biogeografia de Cratylia e áreas prioritárias para coleta. In E.A.Pizarro \& L.Coradin (eds) Potencial del género Cratylia como Leguminosa forrajera. Centro Internacional de Agricultura Tropical - Empresa Brasileira de Pesquisas Agropecuárias, p. 1-28.

SPRENGEL, K. 1825. Systema vegetabilium, ed. 16, 2:463-870. 